\title{
Selenium-Enriched Nickel Selenide Nanosheets as a Robust Electrocatalyst for Hydrogen Generation
}

\author{
Fengmei Wang + Yuanchang Li $i^{+}$Tofik Ahmed Shifa, Kaili Liu, Feng Wang, Zhenxing Wang, \\ Peng Xu, Qisheng Wang, and Jun He*
}

\begin{abstract}
To address the urgent need for clean and sustainable energy, the rapid development of hydrogen-based technologies has started to revolutionize the use of earth-abundant noblemetal-free catalysts for the hydrogen evolution reaction (HER). Like the active sites of hydrogenases, the cation sites of pyrite-type transition-metal dichalcogenides have been suggested to be active in the HER. Herein, we synthesized electrodes based on a Se-enriched NiSe $e_{2}$ nanosheet array and explored the relationship between the anion sites and the improved hydrogen evolution activity through theoretical and experimental studies. The free energy for atomic hydrogen adsorption is much lower on the Se sites $(0.13 \mathrm{eV})$ than on the $\mathrm{Ni}$ sites $(0.87 \mathrm{eV})$. Notably, this electrode benefits from remarkable kinetic properties, with a small overpotential of $117 \mathrm{mV}$ at $10 \mathrm{~mA} \mathrm{~cm}^{-2}$, a low Tafel slope of $32 \mathrm{mV}$ per decade, and excellent stability. Control experiments showed that the efficient conversion of $\mathrm{H}^{+}$into $\mathrm{H}_{2}$ is due to the presence of an excess of selenium in the $\mathrm{NiSe}_{2}$ nanosheet surface.
\end{abstract}

$T_{\text {he global community is facing an unprecedented increase in }}$ energy demand. Aside from the escalating demand scenario, the cleanest way of harnessing/storing energy is an important aspect to be critically considered.$^{[1]}$ Thus tremendous efforts have been devoted to make use of abundant and convenient energy sources, lessening our reliance on fossil fuels. Electrocatalytic water splitting for the generation of hydrogen gas is an environmentally friendly alternative to meet the global energy demands. ${ }^{[2]}$ Platinum group metals are the benchmark catalysts for electrocatalytic hydrogen evolution. ${ }^{[3]}$ However, their high costs and low abundance have hindered their largescale practical application. Thus the discovery of inexpensive electrocatalysts that are based on earth-abundant elements has received significant interest. ${ }^{[4]}$ For example, earth-abundant transition-metal dichalcogenides (TMDs) promote

[*] F. M. Wang, ${ }^{[+]}$T. A. Shifa, K. Liu, F. Wang, Prof. Z. Wang, Prof. J. He CAS Key Laboratory of Nanosystem and Hierarchical Fabrication National Center for Nanoscience and Technology Beijing 100190 (China)

E-mail: hej@nanoctr.cn

F. M. Wang, ${ }^{[+]}$T. A. Shifa, K. Liu, F. Wang

University of Chinese Academy of Sciences Beijing 100049 (P.R. China)

Prof. Y. Li, ${ }^{[+]}$P. Xu, Q. Wang

National Center for Nanoscience and Technology Beijing 100190 (China)

$\left.{ }^{+}\right]$These authors contributed equally to this work.

(4) Supporting information for this article can be found under: http://dx.doi.org/10.1002/anie.201602802. water splitting by catalyzing both the hydrogen evolution reaction (HER) and the oxygen evolution reaction (OER). ${ }^{[2,5]}$ Many two-dimensional layered TMDs, such as $\mathrm{MoS}_{2}{ }^{\left[{ }^{[]}\right.}$and $\mathrm{WS}_{2}{ }^{[7]}$ catalyze the HER in acidic electrolytes. Unfortunately, both density functional theory (DFT) calculations and experimental investigations have confirmed that the superior HER catalytic activity of these materials is derived from the edges rather than the basal planes. ${ }^{[6 a, 8]}$ Furthermore, their low electrical conductivity reduces the catalytic efficiency ${ }^{[9]}$ As a result, other inexpensive TMDs, such as various pyritestructured TMDs $\left(\mathrm{MX}_{2} ; \mathrm{M}=\mathrm{Fe}, \mathrm{Co}\right.$, or $\mathrm{Ni}$ and $\mathrm{X}=\mathrm{S}$ or $\left.\mathrm{Se}\right)$, that are found in minerals or sedimentary deposits ${ }^{[10]}$ have emerged as earth-abundant electrocatalysts with high catalytic activity towards the HER. Some of them, such as $\operatorname{CoS}_{2}$, ${ }^{[11]}$ $\mathrm{CoSe}_{2},{ }^{[12]}$ and $\mathrm{NiSe}_{2},{ }^{[13]}$ benefit from their intrinsic metallic properties. $\mathrm{NiSe}_{2}$ is a Pauli paramagnetic metal with a resistivity below $10^{-3} \Omega \mathrm{cm} \cdot{ }^{[14]}$ The metallicity of $\mathrm{NiSe}_{2}$ makes it use in energy-conversion applications appealing. It has been utilized as an efficient electrocatalyst as a Pt-free counter electrode of dye-sensitized solar cells ${ }^{[15]}$ and as an energy storage material. ${ }^{[16]}$ Admittedly, its potential utilization as a HER catalyst has also been studied, ${ }^{[13]}$ and $\mathrm{NiSe}_{2}$ catalysts were found to perform remarkably well in the HER in acidic electrolytes and demonstrated impressive stability. Previously, the reduced, coordinated surface cations $\left(\mathrm{Ni}^{2+}, \mathrm{Co}^{2+}\right.$, and $\mathrm{Fe}^{2+}$ ), bearing certain similarities to the active centers of hydrogenases, have been suggested to be the active sites of these pyrite electrocatalysts. ${ }^{[10 a, b]}$ Unfortunately, up to now, no direct evidence has been described to support the hypothesis that these sites are responsible for the high catalytic HER activity of $\mathrm{NiSe}_{2}$ catalysts. This information is prerequisite for further catalyst optimization, and the mechanism responsible for the high HER catalytic activity of $\mathrm{NiSe}_{2}$ also needs to be explored.

Beyond addressing these issues, the design of $\mathrm{NiSe}_{2}$ nanomaterials as catalysts for the HER is of great importance. As a representative surface chemical reaction, the HER process is governed by two crucial factors. First, the active sites should be intrinsically active catalytic sites, which is determined by their chemical composition and the interactions between different components. ${ }^{[17]}$ Second, the active sites should be easily accessible to the HER-relevant species (e.g., $\mathrm{H}^{+}, \mathrm{H}_{2}$ ), and the material should thus display good transport properties, which are determined by the specific surface area and the porous structure. For instance, edgeoriented ${ }^{[18]}$ and metallic $\mathrm{MoS}_{2}{ }^{[19]}$ and $\mathrm{WS}_{2}{ }^{[7 \mathrm{a}, 20]}$ are superior HER catalysts. Likewise, it is of great importance to engineer the surface composition and configuration of $\mathrm{NiSe}_{2}$ through controllable synthesis techniques, thereby improving their 
HER performance. Herein, we prepared textured $\mathrm{NiSe}_{2}$ nanosheet arrays on flexible electrodes through selenization. This electrode demonstrated high HER activity and stability in acidic water, with a remarkably low overpotential of $117 \mathrm{mV}$ at $10 \mathrm{~mA} \mathrm{~cm}^{-2}$ and a Tafel slope of $32 \mathrm{mV}$ per decade. Our experiments and DFT calculations corroborate that the Se sites and the $\mathrm{Se}-\mathrm{Se}$ bonds of $\mathrm{NiSe}_{2}$ dominate the striking HER activity on the low-index surface. Furthermore, this selenization method leads to an enrichment of the $\mathrm{NiSe}_{2}$ nanosheet arrays with elemental Se, which is indispensable for facilitating charge transfer and hence improving the catalytic activity.

The vapor selenization technique was utilized to convert ultrathin $\mathrm{Ni}(\mathrm{OH})_{2}$ nanosheets (Supporting Information, Figure $\mathrm{S} 1$ and Note SI) into $\mathrm{NiSe}_{2}$ nanosheets in a quartz socket tube under argon atmosphere (Figure S2). Different products were obtained at different temperatures. The as-prepared samples were then analyzed by scanning electron microscopy (SEM; Figure S3). As seen in Figure 1a, uniform and vertically textured $\mathrm{NiSe}_{2}$ nanosheets covering the entire carbon fibers $(\mathrm{CFs})$ were obtained at $450{ }^{\circ} \mathrm{C}\left(\mathrm{NiSe}_{2}-450\right)$ in a selenium atmosphere. Energy-dispersive X-ray (EDX)

when the reaction temperature is decreased to $400^{\circ} \mathrm{C}$, the obtained nanosheets consist of loosely held nanoparticles $\left(\mathrm{NiSe}_{2}-400\right.$; Figure S4 a). An increase in reaction temperature resulted in the formation of smaller nanoparticles $\left(\mathrm{NiSe}_{2}-500\right.$; Figure S4b). HRTEM imaging and the corresponding selected-area electron diffraction (SAED) pattern in Figure $1 \mathrm{c}$ clearly confirm the high crystallinity of the nanoparticles. The lattice fringes with a spacing of $0.267 \mathrm{~nm}$ are in good agreement with the (210) planes of $\mathrm{NiSe}_{2}$.

The crystal structure of the as-prepared $\mathrm{NiSe}_{2}$ nanosheets was determined by X-ray diffraction (XRD). Aside from the peaks that are due to the CFs (“*”), all other peaks in Figure $1 \mathrm{~d}$ can be indexed to cubic $\mathrm{NiSe}_{2}(a=5.962 \AA$, PDF No. 88-1711). It possesses a pyrite structure in which the $\mathrm{Ni}$ atoms are surrounded by an octahedral arrangement of adjacent Se atoms (Figure 1d, inset). No secondary phase or any other impurity phases were detected, confirming the purity of the product. Furthermore, the reaction temperature has no effect on the crystal type (Figure S5a). Confocal micro-Raman spectra obtained by excitation with an unpolarized $532 \mathrm{~nm}$ laser (Figure 1e and Figure S5b) show four peaks at $148,168,210$, and $239 \mathrm{~cm}^{-1}$ for all $\mathrm{NiSe}_{2}$ samples. The two weak peaks at low energy correspond to the

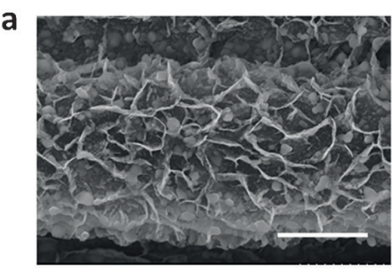

C
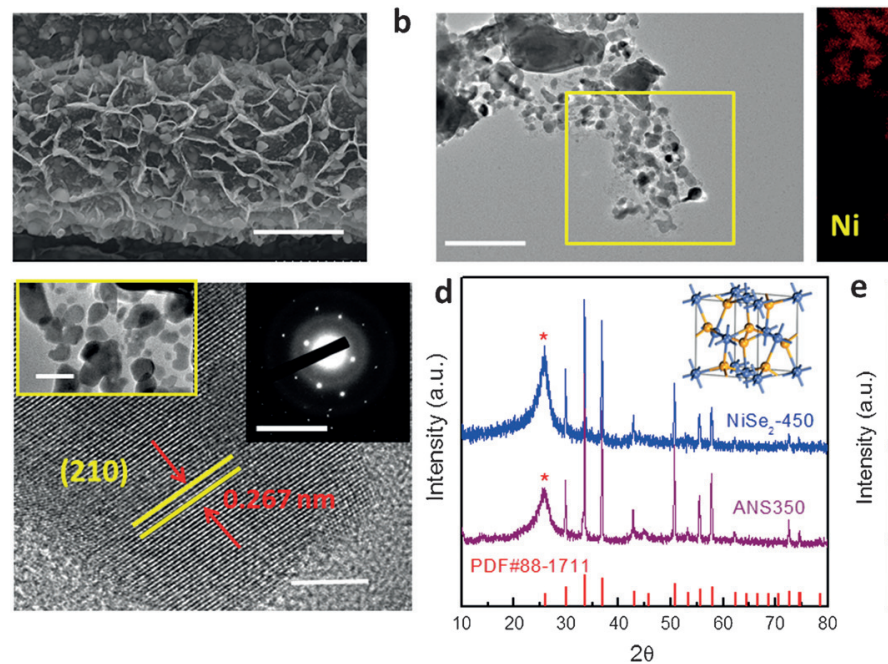

$\mathrm{Ni}$

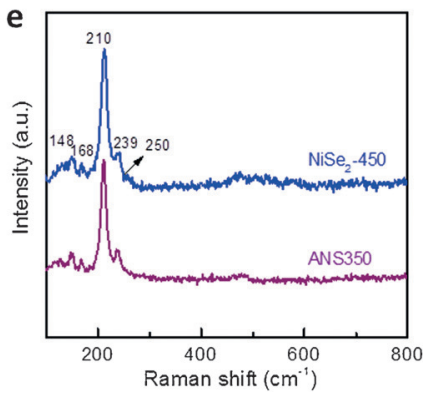

Figure 1. Structural characterization of the $\mathrm{NiSe}_{2}$ nanostructures. a) $\mathrm{SEM}$ image of the as-synthesized $\mathrm{NiSe}_{2}$ 450 nanosheets on CFs. Scale bar: $5 \mu \mathrm{m}$. b) STEM-EDX elemental mapping of the $\mathrm{NiSe}_{2}-450$ nanosheet, showing the homogeneous distribution of $\mathrm{Ni}$ and Se. Scale bar: $200 \mathrm{~nm}$. c) HRTEM image of the $\mathrm{NiSe}_{2}-450$ nanosheets. Insets: Corresponding TEM image (left) and SAED pattern (right). Scale bars: $5 \mathrm{~nm}, 50 \mathrm{~nm}$, and $1 \mathrm{~nm}^{-1}$, respectively. d) XRD patterns of the $\mathrm{NiSe}_{2}-450$ and ANS350 samples compared to that of cubic $\mathrm{NiSe}_{2}$ (PDF No. 88-1711). Inset: Crystal structure of cubic pyrite $\mathrm{NiSe}_{2}$ (Ni cyan, Se yellow). All peaks that are due to the carbon fibers are labeled with "**. e) Raman spectra of the $\mathrm{NiSe}_{2}-450$ and ANS350 nanosheets. The obvious Raman shift at around $250 \mathrm{~cm}^{-1}$ indicates the presence of amorphous selenium. ANS350 refers to an as-grown $\mathrm{NiSe}_{2}-450$ sample further annealed at $350^{\circ} \mathrm{C}$ under argon atmosphere.

elemental mappings (Figure $1 \mathrm{~b}$ ) of $\mathrm{Ni}$ and Se indicate the fairly homogeneous distribution of the constituents across the nanosheet. The morphologies and microstructures of $\mathrm{NiSe}_{2}$ samples prepared at different temperatures were investigated by transmission electron microscopy (TEM) and high-resolution TEM (HRTEM; Figure $1 \mathrm{c}$ and Figure S4). The $\mathrm{NiSe}_{2}-$ 450 nanosheets consist of many loosely bound nanoparticles with sizes of roughly $30 \mathrm{~nm}$ (Figure $1 \mathrm{c}$, inset). By contrast,

\section{As shown in Figure 2 a, th}

XPS spectrum of $\mathrm{NiSe}_{2}-450$ in the $\mathrm{Se} 3 \mathrm{~d}$ region contains an important feature. Selenium atoms in $\mathrm{NiSe}_{2}-450$ possess two valence states. The peaks at higher binding energies $(54.9 \mathrm{eV}$ and $55.7 \mathrm{eV}$ ) could be ascribed to the Se $3 \mathrm{~d}_{5 / 2}$ and $\mathrm{Se} 3 \mathrm{~d}_{3 / 2}$ states of elemental Se, suggesting the presence of elemental Se on the surface. ${ }^{[23]}$ This result is consistent with the Raman

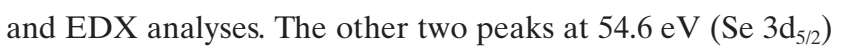

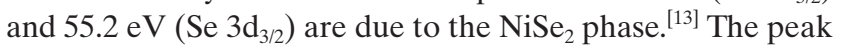



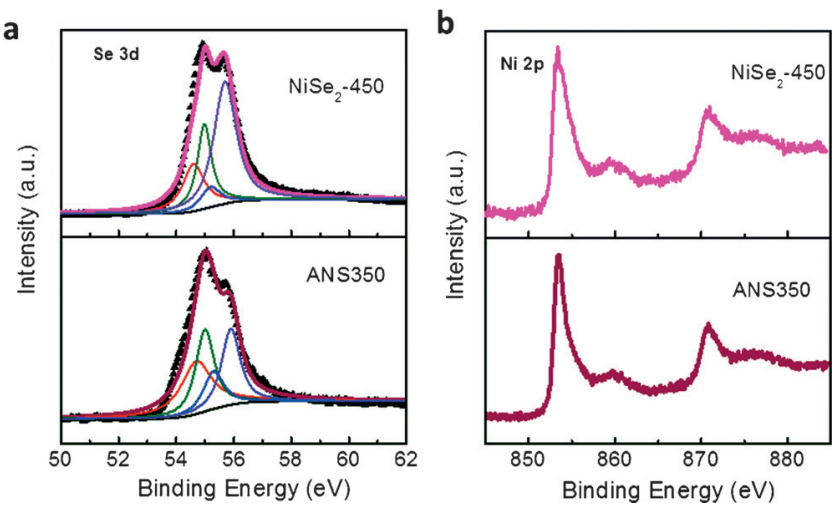

Figure 2. Surface chemical analysis of the different products. a, b) Se $3 \mathrm{~d}$ and $\mathrm{Ni} 2 \mathrm{p}$ regions of the XPS spectra of the $\mathrm{NiSe}_{2}-450$ and ANS350 nanosheets. In (a), the peaks at $54.9 \mathrm{eV}$ (green curve) and $55.7 \mathrm{eV}$ (violet curve) were ascribed to the $\mathrm{Se} 3 \mathrm{~d}_{5 / 2}$ and $\mathrm{Se} 3 \mathrm{~d}_{3 / 2}$ of elemental Se species, suggesting the presence of elemental Se on the surface.

assigned to $\mathrm{Ni} 2 \mathrm{p}_{3 / 2}$ was detected at $853.2 \mathrm{eV}$ (Figure $2 \mathrm{~b}$ and Note SII). These characterizations confirm that a Se-enriched surface is obtained through the selenization reaction in the quartz socket tube. To effectively control the amount of elemental selenium, we annealed the as-grown $\mathrm{NiSe}_{2}-450$ sample at $350^{\circ} \mathrm{C}$ ("ANS350"), which is above the melting point of Se and below the temperature of the reaction, under a flow of argon. ${ }^{[24]}$ After the treatment, the morphology of the nanosheets remained unchanged (Figure S7). Figure 1d reveals that the crystal structure of the as-prepared ANS350 is the same as that of the $\mathrm{NiSe}_{2}-450$ sample. However, its peak at about $250 \mathrm{~cm}^{-1}$ in the Raman spectrum (Figure $1 \mathrm{e}$ ) is inconspicuous. The XPS spectrum of ANS350 (Figure 2b) shows an apparent peak at low binding energy in the Se 3d region, indicating a decrease in the amount of elemental Se on the surface. There are only negligible changes in the XPS spectrum in the Ni $2 p$ region (Figure $2 b$ ). Furthermore, the $\mathrm{Ni} / \mathrm{Se}$ ratio determined by XPS analysis was found to be close to 1:1.5 for ANS350 and 1:3.2 for $\mathrm{NiSe}_{2}-450$. The Se content is clearly significantly lower in ANS350. Therefore, the Raman, EDX, and XPS results unequivocally confirm the presence of elemental Se on the surface of the as-grown $\mathrm{NiSe}_{2}$ nanosheet arrays. Encouraged by the terrific performance and stability of selenium-modified $\mathrm{Ru}$ catalysts in the oxygen reduction reaction in acidic media, ${ }^{[25]}$ we speculated that the elemental $\mathrm{Se}$ on the surface of $\mathrm{NiSe}_{2}-450$ may facilitate hydrogen evolution (see below).

Next, the HER catalytic characteristics of the $\mathrm{NiSe}_{2}$ electrodes were evaluated in $\mathrm{N}_{2}$-saturated $\mathrm{H}_{2} \mathrm{SO}_{4}$ electrolyte and compared to those of platinum foil (see the Supporting Information for details). The ohmic potential drop (iR) losses from the solution resistance were corrected (Figure S8). As seen in Figure $3 \mathrm{a}$, the $\mathrm{CF}$ substrate has a negligible HER performance with a featureless polarization curve. Notably, the Se-rich $\mathrm{NiSe}_{2}-450$ electrode achieved a current density of $10 \mathrm{~mA} \mathrm{~cm}^{-2}$ at a low overpotential of $117 \mathrm{mV}\left(\eta_{10}\right)$, which is lower than those recently reported for other binary pyrite structures. ${ }^{[13,26]}$ This electrode demonstrates the best HER catalytic performance among all synthesized samples, with $\eta_{10}$ values of $125 \mathrm{mV}$ and $129 \mathrm{mV}$ for $\mathrm{NiSe}_{2}-400$ and $\mathrm{NiSe}_{2}-500$, respectively (Figure S9). In an attempt to explore these differences between the as-grown $\mathrm{NiSe}_{2}$ based samples, the double-layer capacitances $\left(C_{\mathrm{dl}}\right)$ were utilized to estimate the effective electrochemically active area of the solid-liquid interface (Note SIII, Figures S10 a-c and $11 \mathrm{a}) .^{[11 \mathrm{a}]}$ The optimized electrochemically active surface area of $\mathrm{NiSe}_{2}-450$, whose $C_{\mathrm{dl}}$ value is 1.7 times than that of $\mathrm{NiSe}_{2}-500$, contributes to the observed superior activity (Table S1). For comparison, a higher overpotential of $152 \mathrm{mV}$ was obtained at the same current density $\left(10 \mathrm{mV} \mathrm{cm}^{-2}\right)$ for the ANS350 electrode in spite of its slightly larger active surface area compared to that of $\mathrm{NiSe}_{2}-450$ (Figure S10d and S11b). Thus, the poorer HER performance of ANS350 can mostly be attributed to the loss of elemental Se from the surface. Figure $3 \mathrm{~b}$ summarizes the overpotentials of different electrocatalysts at 1 and $10 \mathrm{~mA} \mathrm{~cm}^{-2}$. Furthermore, the Se-rich $\mathrm{NiSe}_{2}-450$ nanosheets exhibited a smaller onset potential of approximately $71 \mathrm{mV}$, demonstrating their better catalytic performance compared to ANS350 nanosheets with an onset potential of about $129 \mathrm{mV}$ (Figure S12 and Table S2). These results indicate that all $\mathrm{NiSe}_{2}$ based electrodes are better catalysts than ANS350. Aside from the large surface area, the superior HER activity of $\mathrm{NiSe}_{2}-450$ is believed to arise from the abundant elemental Se on the surface. The corresponding Tafel plots shown in Figure $3 \mathrm{c}$ yield slopes of $32 \mathrm{mVdec}^{-1} \quad\left(\mathrm{NiSe}_{2}-450\right)$ and $31 \mathrm{mV} \mathrm{dec}^{-1}$ (ANS350), which are comparable to that of $\mathrm{Pt}$ foil $\left(31 \mathrm{mV}^{-1}\right)$. These small Tafel slopes for all samples (Figure $3 \mathrm{~d}$ ) indicate a two-electron transfer process following a Volmer-Tafel mechanism of bimolecular adsorption and hydrogen evolution occurring on the surface of these catalysts. This mechanism is consistent with that on Pt. ${ }^{[3 c]}$ To further corroborate the importance of Se enrichment on $\mathrm{NiSe}_{2}-450$, different ANS samples were prepared through reducing the annealing temperature $\left(280\right.$ and $\left.320^{\circ} \mathrm{C}\right)$. Excessive Se could be stripped off from the $\mathrm{NiSe}_{2}$ surface by changing the annealing temperature; thus the amount of surface Se can be controlled without destroying the $\mathrm{NiSe}_{2}$ crystal structure. These ANS280 and ANS320 samples exhibited slightly better catalytic activities for the HER (Figure 3e and Figure S9) than ANS350 in connection to their remnant content of surface Se. Meanwhile, the exchange current density $\left(j_{0}\right)$, the most inherent measure of the HER activity, was carefully determined to assess the quality of the $\mathrm{NiSe}_{2}$ and ANS samples. The $j_{0}$ value of $4.7 \times 10^{-3} \mathrm{~mA} \mathrm{~cm}^{-2}$ for the $\mathrm{NiSe}_{2}-450$ electrode surpasses the value of $1.3 \times$ $10^{-4} \mathrm{~mA} \mathrm{~cm}^{-2}$ for ANS350 as well as the values reported for $\mathrm{NiSe}_{2}$ HER catalysts. ${ }^{[13]}$ In particular, after being normalized by the relative surface area, ${ }^{[11 a]}$ the larger normalized exchange current density $\left(J_{0, \text { normalized }}\right)$ of $\mathrm{NiSe}_{2}-450$ compared to that of ANS350 implies the improved intrinsic activity of the Se-enriched $\mathrm{NiSe}_{2}$ material (Table S1). Electrochemical impedance spectroscopy (EIS) was used to provide further insight into the electrode kinetics of the HER process. The Nyquist plots for $\mathrm{NiSe}_{2}-450$ and ANS350 at an overpotential of $0.1 \mathrm{~V}$ are shown in Figure $3 \mathrm{f}$. Circuit model fitting analysis of the EIS suggests that both electrodes can be modeled using a modified equivalent circuit consisting of a series resistance $\left(R_{\mathrm{s}}\right)$, a constant phase element $(\mathrm{CPE})$, and a charge transfer resistance $\left(R_{\mathrm{ct}}\right)$. The very small observed $R_{\mathrm{ct}}$ value of only 
a

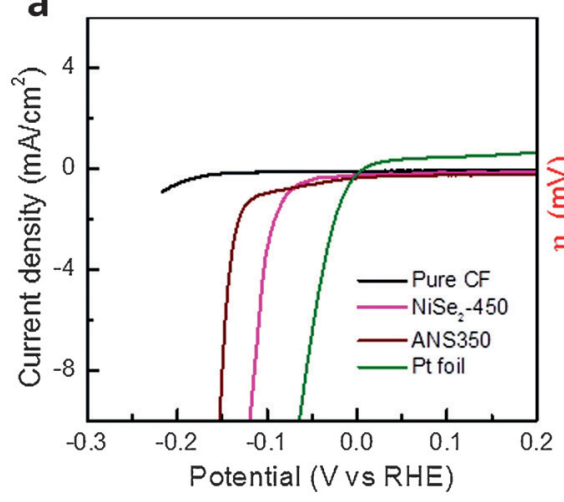

d

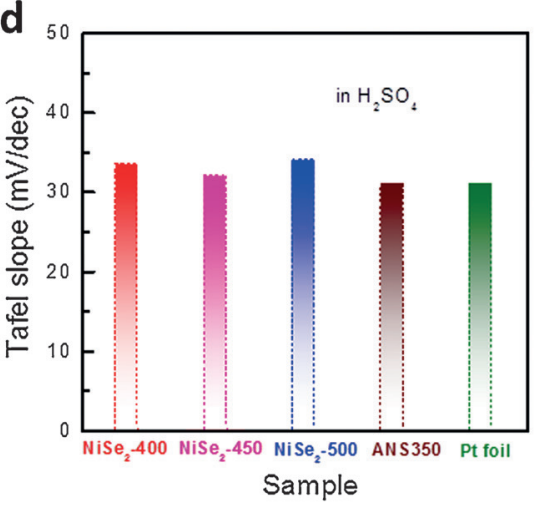

b

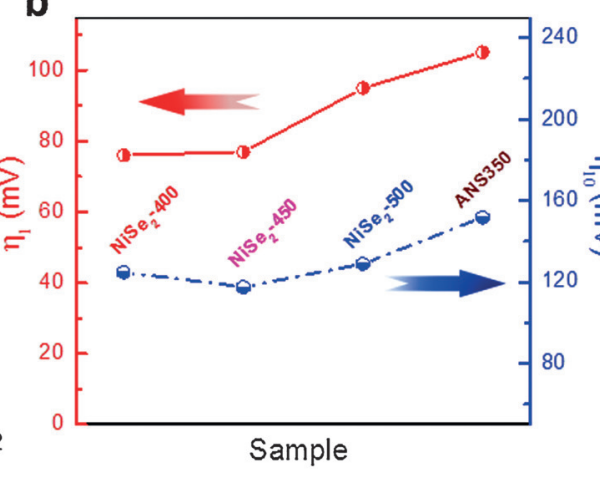

e

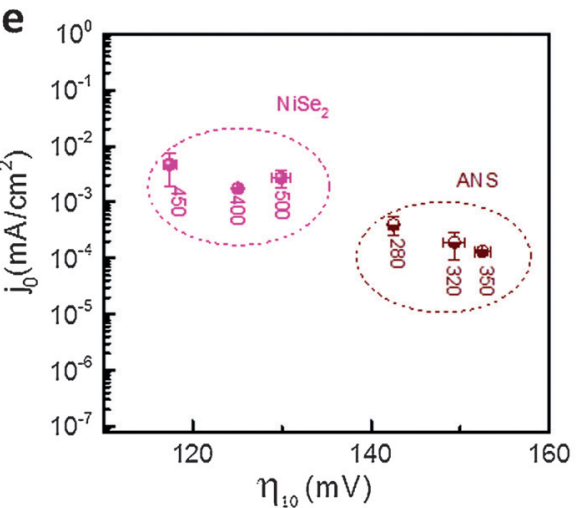

C

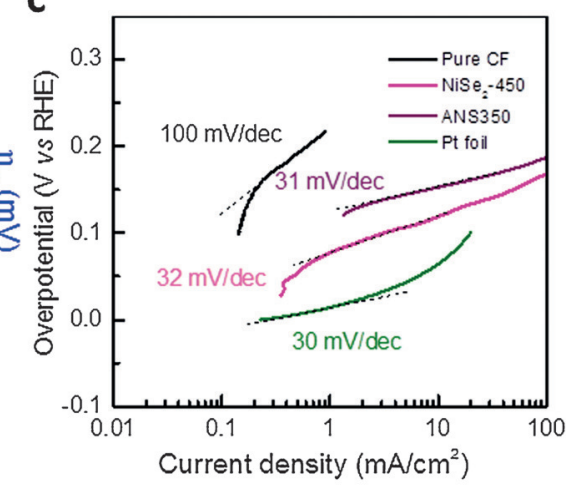

f

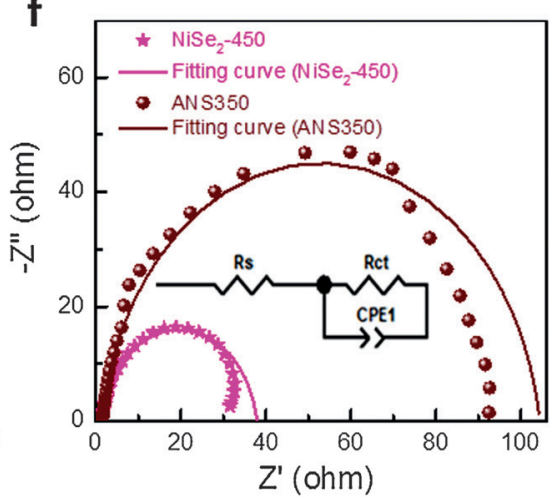

Figure 3. Electrochemical characterization of $\mathrm{NiSe}_{2}$ and ANS nanosheets on carbon fiber electrodes for $\mathrm{HER}$ catalysis. a) Polarization curves for HER with different CF electrodes covered by NiSe ${ }_{2}-450$ and ANS350 nanosheets in $\mathrm{H}_{2} \mathrm{SO}_{4}$ electrolyte $(\mathrm{pH} \approx 0.67)$ in comparison to a Pt foil and pure CF. Sweep rate: $5 \mathrm{mVs}^{-1}$. b) The variation in overpotential at current densities of 1 and $10 \mathrm{mAcm}^{-2}$ obtained from (a). c) Tafel plot for the various catalysts derived from (a). d) Comparison of the Tafel slopes of the different $\mathrm{NiSe}_{2}$ and ANS350 electrodes and Pt foil. e) Statistical summary of the $j_{0}$ values versus the overpotential at $10 \mathrm{mAcm}^{-2}$ for all $\mathrm{NiSe}_{2}$ and ANS electrodes, demonstrating the better $\mathrm{HER}$ activity of $\mathrm{NiSe}_{2}$ electrodes compared to that of ANS electrodes. f) Electrochemical impedance spectroscopy (EIS) Nyquist plots for the NiSe ${ }_{2}-450$ and ANS350 electrodes. The data were fitted using the modified Randles circuits shown in the inset.

$32 \Omega$ for $\mathrm{NiSe}_{2}-450$, which is much smaller than that of $103 \Omega$ for ANS350, indicates a fast Faradic process and thus superior HER kinetics. The high $j_{0}$ value and the small $R_{\mathrm{ct}}$ signal suggest that the elemental Se acts as a promoter, facilitating the charge transfer on the surface to achieve efficient conversion of $\mathrm{H}^{+}$into $\mathrm{H}_{2}$ bubbles. We hypothesize that the interaction and synergy between $\mathrm{NiSe}_{2}$ and $\mathrm{Se}$ is vital to sustain the HER activity. The intrinsic properties of the active sites were shown to be improved when elemental Se modifies the $\mathrm{NiSe}_{2}$ surface, and the interface between them is thus proposed to be the efficient active site in this system. Thus, the established functional link potentially endows the surface with much more pronounced activity for the HER.

Given the high electrocatalytic activity of Se-rich $\mathrm{NiSe}_{2}-$ 450 towards the HER, we further studied its stability to continuously catalyze the generation of $\mathrm{H}_{2}$. After 1000 and 3000 cyclic voltammetry cycles within 6 hours, a negligible decay was observed at high current density, and massive $\mathrm{H}_{2}$ bubbles were generated on the surface of the electrode (Figure $4 \mathrm{a}$ ). The $\mathrm{H}_{2}$ generation can proceed at a constant current density of $-12.5 \mathrm{~mA} \mathrm{~cm}^{-2}$ even over $67 \mathrm{~h}$ of continuous operation (Figure 4b,c, see also Movie S1). The timedependent curve of the $\mathrm{NiSe}_{2}-450$ electrode is of a typical serrate shape (Figure $4 \mathrm{~b}$, inset) because of the alternating processes of bubble accumulation and release. During this process, high-purity nitrogen gas was continuously bubbled into the solution to avoid the accumulation of $\mathrm{H}_{2}$ gas and maintain the $\mathrm{N}_{2}$ saturation. Remarkably, after the stability measurements, XPS studies in the Se $3 d$ and Ni $2 p$ region revealed no obvious changes in the chemical state (Figure $4 \mathrm{~d}, \mathrm{e})$, confirming the robustness of the catalyst. A weak peak around $59 \mathrm{eV}$ in the $\mathrm{Se} 3 \mathrm{~d}$ region indicated that a small amount of $\mathrm{SeO}_{2}$ had been formed on the surface by oxidation in air. The morphology and crystal phase of the sample were almost preserved as confirmed by the SEM images in Figure S13 and the XRD pattern in Figure $4 \mathrm{f}$, respectively.

We further carried out first-principles calculations (see the Supporting Information for details) to evaluate the HER activity of $\mathrm{NiSe}_{2}$. We took the (110) surface as an example as both $\mathrm{Ni}$ and $\mathrm{Se}$ sites can coexist on this surface as shown in Figure $5 \mathrm{a}$. Hydrogen atoms are attached to Se and Ni sites as illustrated in Figure $5 \mathrm{~b}$ and $5 \mathrm{c}$, respectively. Note that there is more than one kind of Se and Ni site for hydrogen adsorption (see Figure S14). Figure $5 \mathrm{~d}$ summarizes the calculated free energies $\left(\Delta G_{\mathrm{H}^{*}}\right)$. The $\mathrm{Se}$ and $\mathrm{Ni}$ sites have free energies of 0.13 and $0.87 \mathrm{eV}$, respectively. Therefore, the Se sites are 

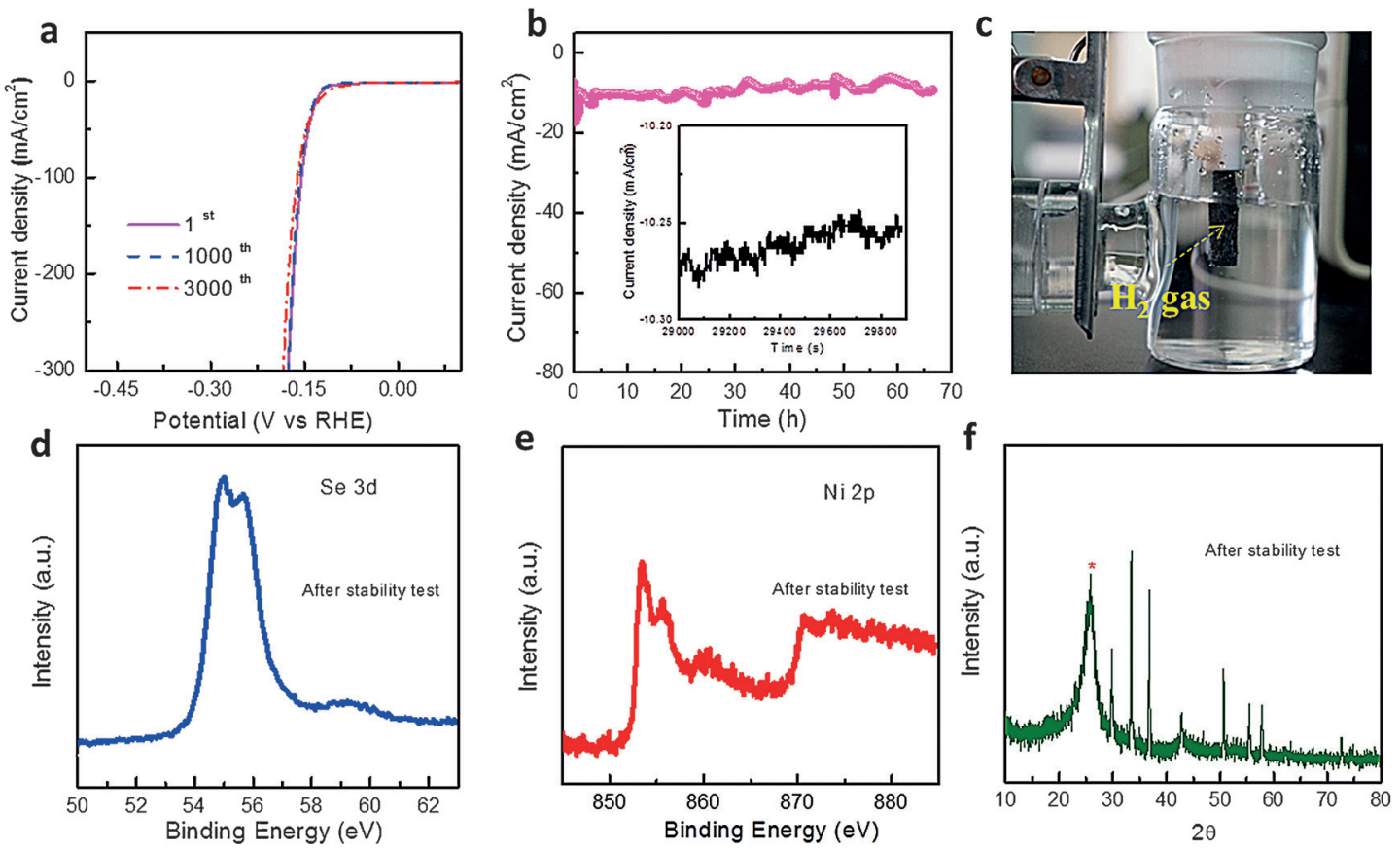

Figure 4. Stability tests for the $\mathrm{NiSe}_{2}-450$ electrode. a) Comparison of the polarization curves after continuous sweeps at $100 \mathrm{mVs}^{-1}$ in acidic $\left(\mathrm{H}_{2} \mathrm{SO}_{4}\right)$ media. b) Time dependence of the current density (about $-12.5 \mathrm{~mA} \mathrm{~cm}^{-2}$ ) during electrolysis for $67 \mathrm{~h}$ at a fixed overpotential of $-150 \mathrm{mV}$. c) Photograph of the process in (b), showing the formation of $\mathrm{H}_{2}$ bubbles on the surface. $d-f$ ) The Se $3 d$ and Ni 2p regions of the XPS spectrum and the XRD pattern of the $\mathrm{NiSe}_{2}-450$ nanosheets after the stability test, suggesting that no obvious changes had occurred.
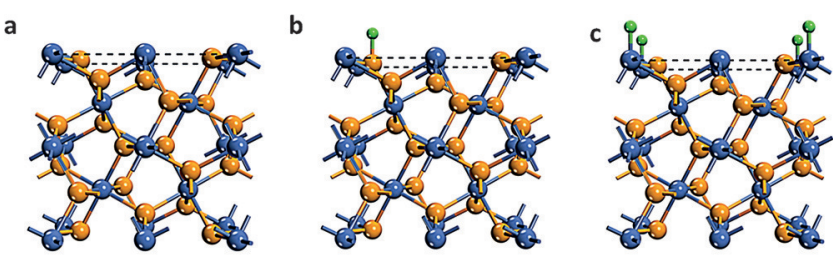

d
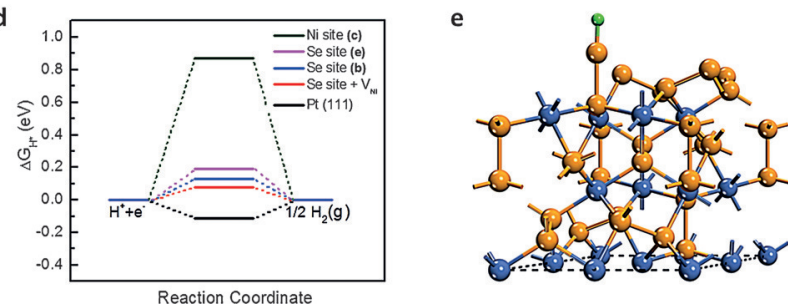

Figure 5. Free-energy diagram from first-principles calculations. a) Slab model of the $\mathrm{NiSe}_{2}$ (110) surface, where both $\mathrm{Ni}$ and Se atoms are present in the termination layer. Hydrogen adsorption at b) Se sites and c) Ni sites. d) Free energy $\left(\Delta G_{H^{*}}\right)$ diagram for different hydrogenadsorbed states. e) Hydrogen adsorption at a Se-terminated (111) surface. In (d), the red line corresponds to the configuration with a surface $\mathrm{Ni}$ (four-coordinated) atom removed from (b). $\mathrm{H}$ green, $\mathrm{Ni}$ cyan, Se yellow. The black dashed rhomboids denote the surface unit cells.

responsible for the good catalytic activity of $\mathrm{NiSe}_{2}$, but not the previously assumed Ni sites. ${ }^{[10 a, b, 12]}$ In our experiment, the Seenriched $\mathrm{NiSe}_{2}-450$ sample, with a $\mathrm{Ni} / \mathrm{Se}$ ratio of $1: 3.2$, exhibited the best performance. To mimic such a Se-rich environment, we removed one $\mathrm{Ni}$ atom on the terminated surface and then calculated the free energy for hydrogen adsorption on the same Se site. It was found that the free energy becomes $0.08 \mathrm{eV}$, approaching zero. On the other hand, a Se-rich environment may arise from the adsorption of excess $\mathrm{Se}$ on $\mathrm{NiSe}_{2}$ surface. Figure 5 e shows a Se-terminated (111) surface, in which the topmost Se atom only binds to another Se atom underneath. We expect that such a model can partially describe the Se adsorption case. It gives a free energy of $0.19 \mathrm{eV}$. Note, however, that such a Se atom is unstable for the adsorption of more that one hydrogen atom, even if it has a better free energy of $0.02 \mathrm{eV}$. All of these results suggest that the Se centers are the active sites for the HER rather than the Ni sites in $\mathrm{NiSe}_{2}$. Whereas excess Se on the surface can considerably improve the overall performance, elemental Se particles would suffer from low stability. Therefore, careful interface engineering is necessary to achieve a good balance between HER activity and stability.

In summary, we have presented selenium-enriched pyritetype $\mathrm{NiSe}_{2}$ as an efficient high-performance and earthabundant catalyst for robust electrochemical hydrogen evolution. Aside from the large accessible surface area, the excess selenium on the $\mathrm{NiSe}_{2}$ surface accelerates the changer transfer during the HER process as they optimize the intrinsic catalytic capacity of the $\mathrm{NiSe}_{2}$ nanosheets to a certain extent and act as promoters for $\mathrm{H}_{2}$ production. In combination with DFT calculations, we showed that the Se sites and the $\mathrm{Se}-\mathrm{Se}$ bonds of $\mathrm{NiSe}_{2}$ are of critical importance for the striking HER activity and stability. 


\section{Acknowledgements}

This work was supported by the National Natural Science Foundation of China (21373065 and 61474033), the 973 Program of the Ministry of Science and Technology of China (2012CB934103), the Beijing Natural Science Foundation (2144059), and CAS Key Laboratory of Nanosystem and Hierarchical Fabrication. We gratefully thank the K. C. Wong Education Foundation for support.

Keywords: chalcogenides - electrocatalysis .

hydrogen evolution reaction $\cdot$ nickel $\cdot$ selenium

How to cite: Angew. Chem. Int. Ed. 2016, 55, 6919-6924 Angew. Chem. 2016, 128, 7033-7038

[1] a) J. A. Turner, Science 2004, 305, 5686; b) M. Chhowalla, H. S Shin, G. Eda, L.-J. Li, K. P. Loh, H. Zhang, Nat. Chem. 2013, 5 , 263-275; c) M. Grätzel, Nature 2001, 414, 338-344; d) Y. Wang, Q. Wang, X. Zhan, F. Wang, M. Safdar, J. He, Nanoscale 2013, 5, $8326-8339$.

[2] F. Wang, T. A. Shifa, X. Zhan, Y. Huang, K. Liu, Z. Cheng, C. Jiang, J. He, Nanoscale 2015, 7, 19764-19788.

[3] a) J. Greeley, T. F. Jaramillo, J. Bonde, I. Chorkendorff, J. K. Nørskov, Nat. Mater. 2006, 5, 909-913; b) B. Hinnemann, P. G. Moses, J. Bonde, K. P. Jørgensen, J. H. Nielsen, S. Horch, I. Chorkendorff, J. K. Nørskov, J. Am. Chem. Soc. 2005, 127, $5308-$ 5309 ; c) B. E. Conway, B. V. Tilak, Electrochim. Acta 2002, 47, 3571-3594; d) Z. Fan, Z. Luo, X. Huang, B. Li, Y. Chen, J. Wang, Y. Hu, H. Zhang, J. Am. Chem. Soc. 2016, 138, 1414-1419.

[4] M. S. Faber, S. Jin, Energy Environ. Sci. 2014, 7, 3519-3542.

[5] a) Q. Lu, Y. Yu, Q. Ma, B. Chen, H. Zhang, Adv. Mater. 2016, 28, 1917-1933; b) J. Miao, F. X. Xiao, H. B. Yang, S. Y. Khoo, J. Chen, Z. Fan, Y.-Y. Hsu, H. M. Chen, H. Zhang, B. Liu, Sci. Adv. 2015, 1, e1500259; c) J. Chen, X. J. Wu, L. Yin, B. Li, X. Hong, Z Fan, B. Chen, C. Xue, H. Zhang, Angew. Chem. Int. Ed. 2015, 54 1210-1214; Angew. Chem. 2015, 127, 1226-1230; d) C. B. Ma X. Qi, B. Chen, S. Bao, Z. Yin, X. J. Wu, Z. Luo, J. Wei, H. L. Zhang, H. Zhang, Nanoscale 2014, 6, 5624-5629; e) Z. Zeng, C Tan, X. Huang, S. Bao, H. Zhang, Energy Environ. Sci. 2014, 7 797 -803; f) X. Huang, Z. Zeng, S. Bao, M. Wang, X. Qi, Z. Fan, H. Zhang, Nat. Commun. 2013, 4, 1444.

[6] a) T. F. Jaramillo, K. P. Jørgensen, J. Bonde, J. H. Nielsen, S. Horch, I. Chorkendorff, Science 2007, 317, 100-102; b) J. V. Lauritsen, J. Kibsgaard, S. Helveg, H. Topsoe, B. S. Clausen, E. Laegsgaard, F. Besenbacher, Nat. Nanotechnol. 2007, 2, 53-58; c) Y. Tan, P. Liu, L. Chen, W. Cong, Y. Ito, J. Han, X. Guo, Z. Tang, T. Fujita, A. Hirata, M. W. Chen, Adv. Mater. 2014, 26, 8023 -8028; d) Y. Yan, B. Xia, Z. Xu, X. Wang, ACS Catal. 2014, 4, 1693-1705; e) H. Li, C. Tsai, A. L. Koh, L. Cai, A.W. Contryman, A. H. Fragapane, J. Zhao, H. S. Han, H. C. Manoharan, F. Abild-Pedersen, J. K. Norskov, X. Zheng, Nat. Mater. 2016, 15, 48-53

[7] a) D. Voiry, H. Yamaguchi, J. Li, R. Silva, D. C. B. Alves, T. Fujita, M. Chen, T. Asefa, V. B. Shenoy, G. Eda, M. Chhowalla, Nat. Mater. 2013, 12, 850-855; b) K. Xu, F. Wang, Z. Wang, X. Zhan, Q. Wang, Z. Cheng, M. Safdar, J. He, ACS Nano 2014, 8, $8468-8476$; c) Y. Jung, J. Shen, Y. Liu, J. M. Woods, Y. Sun, J. J. Cha, Nano Lett. 2014, 14, 6842-6849.
[8] A. B. Laursen, S. Kegnaes, S. Dahl, I. Chorkendorff, Energy Environ. Sci. 2012, 5, 5577-5591.

[9] a) X. Zou, Y. Zhang, Chem. Soc. Rev. 2015, 44, 5148-5180; b) Y. Li, H. Wang, L. Xie, Y. Liang, G. Hong, H. Dai, J. Am. Chem. Soc. 2011, 133, 7296-7299.

[10] a) D. Kong, J. J. Cha, H. Wang, H. R. Lee, Y. Cui, Energy Environ. Sci. 2013, 6, 3553-3558; b) M. Cabán-Acevedo, M. L. Stone, J. R. Schmidt, J. G. Thomas, Q. Ding, H.-C. Chang, M.-L. Tsai, J.-H. He, S. Jin, Nat. Mater. 2015, 14, $1245-1251$; c) M. S. Faber, M. A. Lukowski, Q. Ding, N. S. Kaiser, S. Jin, J. Phys. Chem. C 2014, 118, 21347-21356; d) X. Wu, B. Yang, Z. Li, L. Lei, X. Zhang, RSC Adv. 2015, 5, 32976-32982; e) H. Zhang, B. Yang, X. Wu, Z. Li, L. Lei, X. Zhang, ACS Appl. Mater. Interfaces 2015, 7, 1772-1779.

[11] a) M. S. Faber, R. Dziedzic, M. A. Lukowski, N. S. Kaiser, Q. Ding, S. Jin, J. Am. Chem. Soc. 2014, 136, 10053-10061; b) S. Peng, L. Li, X. Han, W. Sun, M. Srinivasan, S. G. Mhaisalkar, F. Cheng, Q. Yan, J. Chen, S. Ramakrishna, Angew. Chem. Int. Ed. 2014, 53, 12594-12599; Angew. Chem. 2014, 126, $12802-12807$.

[12] D. Kong, H. Wang, Z. Lu, Y. Cui, J. Am. Chem. Soc. 2014, 136, 4897-4900.

[13] J. Liang, Y. Yang, J. Zhang, J. Wu, P. Dong, J. Yuan, G. Zhang, J. Lou, Nanoscale 2015, 7, 14813-14816.

[14] M.-Z. Xue, Z.-W. Fu, Electrochem. Commun. 2006, 8, 1855 1862.

[15] F. Gong, X. Xu, Z. Li, G. Zhou, Z. S. Wang, Chem. Commun. 2013, 49, $1437-1439$.

[16] J. A. Reyes-Retana, G. G. Naumis, F. Cervantes-Sodi, J. Phys. Chem. C 2014, 118, 3295-3304.

[17] J. Zhuo, M. Cabán-Acevedo, H. Liang, L. Samad, Q. Ding, Y. Fu, M. Li, S. Jin, ACS Catal. 2015, 5, 6355-6361.

[18] a) M. R. Gao, M. K. Chan, Y. Sun, Nat. Commun. 2015, 6, 7493; b) D. Kong, H. Wang, J. J. Cha, M. Pasta, K. J. Koski, J. Yao, Y. Cui, Nano Lett. 2013, 13, 1341-1347.

[19] a) D. Voiry, M. Salehi, R. Silva, T. Fujita, M. Chen, T. Asefa, V. B. Shenoy, G. Eda, M. Chhowalla, Nano Lett. 2013, 13, 6222 6227 ; b) M. A. Lukowski, A. S. Daniel, F. Meng, A. Forticaux, L. Li, S. Jin, J. Am. Chem. Soc. 2013, 135, 10274-10277.

[20] F. Wang, J. Li, F. Wang, T. A. Shifa, Z. Cheng, Z. Wang, K. Xu, X. Zhan, Q. Wang, Y. Huang, C. Jiang, J. He, Adv. Funct. Mater. 2015, 25, 6077-6083.

[21] H. Fan, M. Zhang, X. Zhang, Y. Qian, J. Cryst. Growth 2009, 311, $4530-4534$

[22] a) X. Zhou, P. Gao, S. Sun, D. Bao, Y. Wang, X. Li, T. Wu, Y. Chen, P. Yang, Chem. Mater. 2015, 27, 6730-6736; b) G. W. Tang, Q. Qian, K. L. Peng, X. Wen, G. X. Zhou, M. Sun, X. D. Chen, Z. M. Yang, AIP Adv. 2015, 5, 027113.

[23] C. Zhang, H. Tao, Y. Dai, X. He, K. Zhang, Prog. Nat. Sci.: Mater. 2014, 24, 671-675.

[24] G. Zehl, G. Schmithals, A. Hoell, S. Haas, C. Hartnig, I. Dorbandt, P. Bogdanoff, S. Fiechter, Angew. Chem. Int. Ed. 2007, 46, 7311-7314; Angew. Chem. 2007, 119, 7452-7455.

[25] a) P. K. Babu, A. Lewera, J. H. Chung, R. Hunger, W. Jaegermann, N. Alonso-Vante, A. Wieckowski, E. Oldfield, J. Am. Chem. Soc. 2007, 129, 15140-15141; b) D. Cao, A. Wieckowski, J. Inukai, N. Alonso-Vante, J. Electrochem. Soc. 2006, 153, A869.

[26] H. Liang, L. Li, F. Meng, L. Dang, J. Zhuo, A. Forticaux, Z. Wang, S. Jin, Chem. Mater. 2015, 27, 5702-5711.

Received: March 20, 2016

Published online: April 25, 2016 